\title{
The Research on Live Surveillance Monitoring Technology for Marketing Expenses Remote Control
}

\author{
Shuiping Dinga ${ }^{a}$, Haifeng Lin ${ }^{b}$, Guang Chen ${ }^{c}$, Xiaoguang Li ${ }^{d}$ \\ Beijing Kedong Electric Power Control System co., LTD, Beijing, 100192, China \\ adingshuiping@sgepri.sgcc.com.cn, blinhaifeng@sgepri.sgcc.com.cn, cchengguang@163.com, \\ dlixiaoguang_kd@163.com
}

Keywords: mass data storage, distributed database, comparison test

\begin{abstract}
Electricity Cost Remote Control in Marketing Business has been playing an important role in the business management of electricity information collection. Through analysis on the information about electricity cost remote control, it can study the cost control data and provide the real-time estimation on energy consumption so as to establish the interaction with the customers and put forward the remote control mode by analyzing the data processing from data classification, storage, distribution and circulation, which will be greatly improved electricity information collection system.
\end{abstract}

\section{Introduction}

The application of live surveillance monitoring technology for marketing expenses remote control consists of benchmark strategy management, power electricity measurement, balance automatic alarm, remote electricity control and so on. According to the electric energy data collected automatically, the real-time calculation of electricity is adopted.

Live surveillance monitoring technology for marketing expenses remote control has achieved certain results in business development. However, in the application of real-time measurement and remote control, it still lacks monitoring and analysis on the implementation process and business data which make the control unable to effectively support the whole process of business management. If the fee control customer and relevant data can be applied to conduct a comprehensive multi dimension control and analysis, it can have a large role in promoting the customer service level and innovative management.

Remote real-time marketing cost control is based on the real-time monitoring and analysis of charge control data processing. The research is about the multi-dimensional graphical monitoring technology with which the key data of monitoring can be processed, and it can service for the application mode of remote real-time fee control.

\section{Work of live surveillance monitoring technology for marketing expenses}

Marketing remote real time cost control application operation analysis technology is becoming mature. And the monitoring technology has also been gradually developed. According to the comprehensive analysis of the application of the standardized design of marketing remote real-time fee control, the cost control work is mainly launched from three aspects.

\subsection{Business real-time monitoring}

The monitoring and analysis are adopted in data collection, electricity tariff calculation, cost control implementation, purchasing power, etc. Through the analysis of early warning, power failure, complex operation, SMS notification operation, Bill send operation, users' development, affecting the cost, revenue enhancement, the results of analysis can be achieved. At the same time, the analysis results are checked to determine the cost control business to get real-time monitoring. The time and space are no longer restricted, and a multi business level monitoring application can be achieved. 


\subsection{Operation of inspection management}

Check the cost control strategy and the quality of terminal equipment. Through developing inspection tasks, assigning tasks to the site or remotely collecting data from the main station, the analysis and review of the inspection management can be realized. Inspection is required to complete baseline verification, data set back copy testing, remote control test and long-term payment records user verification in order to realize the marketing cost control remote real-time monitoring technology.

\subsection{Data mining and analysis}

According to the cost control execution of business data and customer purchase data, the data mining and analysis, including frequency, amount and interval are executed. It can be used as a basis for cost control strategy formulation, customer base division, business development and promotion. Data mining and analysis need to complete the benchmark threshold implementation situation analysis, reference threshold simulation analysis, cost control threshold and customer payment analysis and customer payment analysis.

\section{Live surveillance monitoring technology for marketing expenses remote control}

The overall technical threshold of the application of remote control analysis and monitoring is relatively low. The technical difficulty are in data mining and analysis. And based on the analysis of the application results of multi-dimension graphical monitoring technology, it can improve the application of marketing remote real-time cost control monitoring technology.

\subsection{Multi-dimensional data analysis and display technology}

Multidimensional data analysis and display technology provides technical support for some areas, such as the application of process cost control monitoring and analysis, control strategy assessment, etc. Its application can be gradually extended

Multidimensional data analysis and display technology can be achieved through two aspects:

(1) Information is integrated and based on real-time data, statistical data mining and analysis are proposed.

(2) The multidimensional data analysis model is established, and it can realize the dynamic real-time display of analysis results.

\subsection{Real-time measurement and statistics of cost control}

Cost control real-time measurement and statistics techniques will be used for the cost control of real-time measurement results, which can service for month end settlement and report statistics providing theoretical and technical support. The realization of these technologies can support the two deepening of the application of cost control. The data collected by the electric information system acquisition terminal is measured in real time. Then analyze the statistical data of the statements and unearth sufficient information under the data which can be used again.

\subsection{Customer oriented real time interactive remote cost control application technology}

The construction of real-time interactive remote cost control application technology for customers can meet the goal of cost control real-time interaction and efficient operation requirements.

\section{Marketing remote real time data processing}

Cost control business related monitoring and analysis, is to provide business and technical support for the realization of cost control business management and control. In the process of marketing remote real-time cost control monitoring technology, customer oriented real-time interactive remote fee control application technology is difficult to realize. Through the multi-dimensional data mining and analysis, a real time interactive remote cost control application technology for customers is built to meet the cost control real-time interaction and efficient operation requirements. 


\subsection{Data classification}

In the process of storage, calculation and transmission, the different types of data have different technology to realize, so it is necessary to classify the data, and then realize the classification management.

According to the principles of China State Grid Corp data classification, marketing remote real-time fee control application data is divided into two levels. The first layer is structured data, unstructured data and massive quasi real time data. The structure data of the second layer is composed of metadata, its coding and service data. Unstructured data is composed of documents. The mass quasi real time data is composed of service data.

\subsection{Data transfer}

Marketing remote real time charge control application will exchange data with marketing business application and electric energy collection system. Marketing remote real time cost control application gets the basic file data from the marketing business application and gets the meter reading data form the electric energy collection system. After the calculation results of the electricity generation, marketing remote real-time charge control application will push fee control request to marketing business application system. Data transfer between marketing remote real time charge control application and applications are shown in Figure 1.

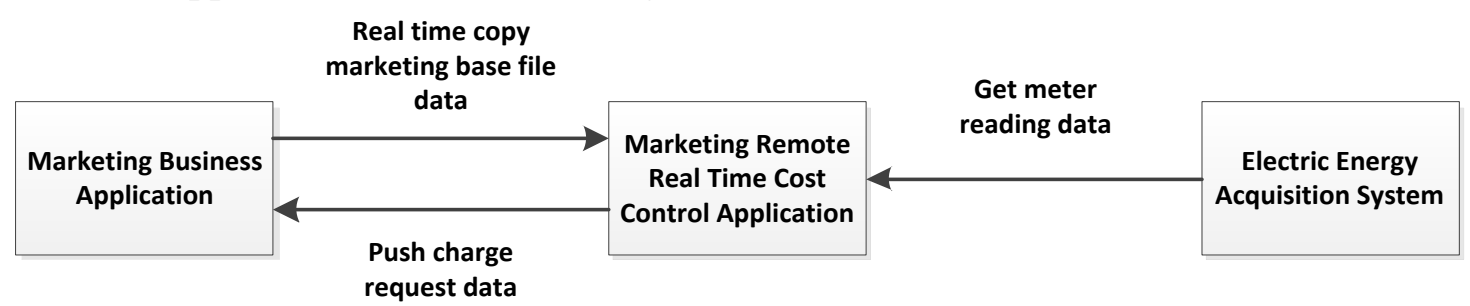

Fig.1 Data flow diagram

Remote real-time marketing cost control applications involving data transfer and storage area is divided into cost control production base, buffer, data exchange area, engine of data loading, data back to writing engine, real-time calculation engine, request push engine etc. Through marketing business application and the data obtained from electric energy acquisition system, the data support is provided to the marketing remote real time cost control application.

Through the database replication technology, the marketing production base of customers, billing files, billing parameters and account balances are synchronized to cost control production base which can provide customer profile data for the application of remote real time cost control in marketing.

The basic data used for calculating the required basic data, is pre-loaded into the cache area, which is based on the calculation of the necessary basic data, such as electric customers, charging files, charging parameters, meter reading data, strategy application, account balance, charge control strategy and so on.

Through the Middle Library, the electric information acquisition system provides a number of meter reading data for marketing remote real-time charge control applications. Real time calculation engine reads the meter data from the middle library. The number of meter reading is analyzed and organized as the standard format of the meter reading data, directly loaded into the calculation of the calculation of the program memory. When the meter reading data is loaded into the calculation of memory, the implementation of the calculation program begins. According to the meter reading data, calculation process obtains customer information file data, and then calculate the memory. Finally, and then to the cost control production base to write the results. Finally, the results of the calculation of the cost control will be written back to the library.

The data classification and data transfer process of remote real time cost control in marketing is analyzed. According to the remote real-time marketing cost control application data transfer and processing mode, it is divided into marketing data synchronization, marketing data loading, data acquisition receiving, measuring results back writing, costs control request pushing. 
If the marketing infrastructure data platform can provide services to the outside, the subsequent basis of the file data is no longer replicated from the marketing database, but the basic data platform for marketing.

\subsection{Data storage and distribution}

According to the data classification of the marketing remote real-time fee control application, combined with the system application architecture design, marketing business application and the construction of electrical information collection system, the data storage and distribution of marketing remote real time cost control application are scheduled.

This system involves only two parts: the structured data and unstructured data. Structured data storage is distributed in the marketing production base, cost control production base and buffer area. Mass quasi real time data and non-structured data are distributed in the data exchange area. The fee control strategy data and abnormal data are stored in the charge control business, and this part of the data is derived from the charge control production base. Customer information file data, meter reading data, marketing standard specification coding data, electricity tariff calculation and system operation of the basic data are stored in the cost control production base. The calculation result data, cost control strategy data and abnormal data are also stored in the cost control production base. The cache area stores the data of pre-loaded customer information file data, the standard of marketing the data of charge control strategy and so on. Data exchange area storages copy number file set and measurement results file set.

\section{Marketing remote real time fee control application monitoring technology application mode}

Based on the SG186 standardization design results, marketing remote real-time fee control application is generally in the calculation process, real-time accessing to database query to calculate the user profile information data for meter reading data. After the calculation is completed, the results of the calculation in real time are back to the database. This implementation model is estimated to calculate the capacity of about 1 million -200 million per hour. The main performance bottleneck lies in the user profile information acquisition, data acquisition and results back writing link.

Based on the existing tariff calculation technology framework, the user profile information data is copied from the marketing database to the fee control database, and the existing tariff calculation program structure is maintained. This can meet the some cities' requirements of electricity tariff calculation of which has the number of 400 million cost control users. This mode of marketing remote real-time control of the typical architecture is as follows fig.2.

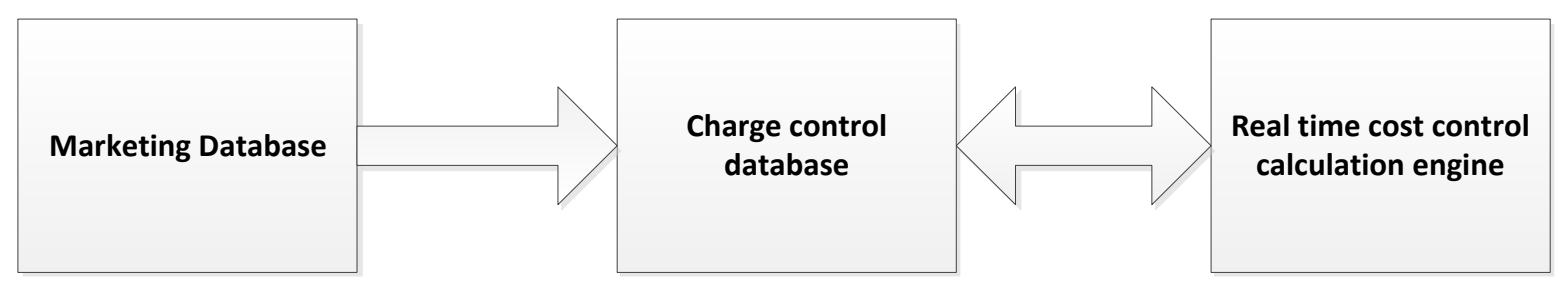

Fig. 2 This mode of marketing remote real-time control

\section{Conclusion}

When the province's cost control user size is below 4 million, the existing tariff calculation architecture can meet the requirements of carrying out the cost control business. If the fee charged subscribers reached more than 400 million, the existed electricity computing architecture technology needs upgrading and user profile data acquisition, meter reading data access link and the calculation result back writing link needs optimizing. It can make contributions to the improvement of tariff calculation ability and marketing business requirements. As an important part of the marketing business management, the application of the remote fee control system has been effectively improved, whether it is from the aspect of electricity tariff risk technology or management. Through the deep 
analysis of the data of real time charge control, the power flow and information flow with customers not only enhance customer service capabilities, it is also a great improvement in technology. In addition, it has great significance to the second use of the remote control system.

\section{References}

[1]. Chongqing Li. The application about electricity cost remote control in marketing business, Power Demand Side Management, Vol. 17(2015) No.4.

[2]. Zhenhong $\mathrm{Xu}$. Design of intelligent electric meter based on remote cost control, China High-Tech Enterprises, No.29 (2013).

[3]. Yufeng Zhang. Application of intelligent charge control system, East China Science \& Technology, 2014(9).

[4]. Nvgui Lin, Sangbi Mo. Study on the Power Supply Enterprise's Selection on the Smart Electric Meter, Journal of Minjiang University, Vol. 34 (2013) No. 1,pp40-43.

[5]. Peng He. Research on the application of intelligent electric energy meter in remote charge control, China Science and Technology Review, 2011(32).

[6]. Jian Zhang. Research on the optimization scheme of intelligent electric energy meter remote cost control execution process, SILICON VALLEY, 2014(18). 